\title{
Editorial
}

\section{Statistical models and applications in biomedicine}

\author{
"You cannot escape the statistical method, so you may as well make friends with it." - Josiah Stamp, Some \\ Economic Factors in Modern Life [1]
}

"Approximately half the articles published in medical journals that use statistical methods use them incorrectly."S.A. Glantz, Biostatistics: How to Detect, Correct, and Prevent Errors in Medical Literature [1]

This issue is the first issue of Model Assisted Statistics and Applications devoted to biomedicine. Statistical methodology is a key component of modern medical research and is an essential element in both the academic and regulatory setting. As the comments by Glantz imply, good research requires more than using statistical methods but using statistical methods correctly. So statisticians have an ongoing role to play in advancing biomedicine.

In this issue one finds a range of problems and statistical solutions.

For example, three papers ( $\mathrm{Lu}, \mathrm{Li}$, and Soon; Chen and Li; Nie, Soon, Tauber, and Huque) describe issues that can occur when evaluating clinical trials. Few clinical trials today are focused on a single continuous endpoint. Lu et al. focus on an issue of multiplicity whereas Chen and Li focuses on a situation where not all values of the continuous outcome variable have relevant clinical meaning. Non-inferiority trials are common in a regulatory setting where it is unethical to use a placebo treatment arm but one is still concerned about establishing a new drug is effective and not that much worse than a drug approved for the same indications. Nie et al. note that the patient mix can vary among trials and the authors provide a modeling approach for adjusting for patient mix in the context of a non-inferiority trial.

The paper by Song and Weissfeld deal with comparing two groups of patients with time to event data (e.g. time to relapse in already diagnosed patients) but in the context of clinical studies that are not randomized, e.g. in observational studies in which one agrees to enroll patients already in the clinic for varying amounts of time and then accrue new patients as they come in. The already enrolled patients tend to be non-representative in that patients on board the longest tend to be overrepresented; the issue of how to compare groups in the presence of such length bias is addressed in the manuscript. Two of the papers (Pahwa and Senthilselvan; Begum, Dwivedi, Pandey, and Mittal) deal with epidemiological applications where hierarchical and/or correlated structures are common. In one instance (Pahwa and Senthilselvan), correlation comes from repeatedly assessing the same patient. Some of the data may be censored and the authors present an approach to deal with clustered survival data to address assessment of bronchial responsiveness. In the other instance, Begum et al. provide an analysis of unwanted pregnancies using a hierarchical model with a binary response using a mixed model approach. The use of random effects in the model generates a correlation structure that considers women within a state to be more correlated than women from different states.

The paper by Zaslavsky, Han, Tiwari, and Puri is representative of the decade plus rise in interest in genomics in modern medical research and provides an alternative approach to analyzing microarray data in the context of big $p$ (lots of features) and small $n$ (numbers of replicates). The goal is to find differentially expressed genes between groups or interest.

The paper by Blodgett examines lack of fit metrics for serial dilution experiments. These methods are often used by clinical and food microbiologists to obtain quantitative estimates of microbial density from heterogeneous samples (e.g. meat or fecal samples). 
These papers span a small fraction of the possible statistical topics one finds in biomedical applications but hopefully this set will intrigue the readers of MASA.

Estelle Russek-Cohen, Ph.D. U.S. FDA and Boris Zaslavsky, Ph.D., Dr.Sc. U.S. FDA.

\section{Reference}

[1] C.C. Gaither and A. A. Cavazos-Gaither. Statistically Speaking. IOP Publishing Ltd., Bristol U.K. and Philadelphia, PA., 1996. 